\title{
PARASITOSES OPPORTUNISTES EN AFRIQUE. CLINIQUE ET DIAGNOSTIC
}

\author{
B. KAPITA*, R. COLEBUNDERS*, K. LUSAKUMUNU*, M. C. HENRY**
}

\begin{abstract}
RÉSUMÉ
Les parasitoses opportunistes sont de plus en plus diagnostiquées chez les patients SIDA africains. Les prévalences actuelles révèlent que la cryptosporidiose est la parasitose la plus fréquente avec une prévalence de 7 à $31 \%$. L'Isospora belli a une fréquence de 5 à $19 \%$ suivant les auteurs. Ces parasitoses sont probablement une des causes de diarrhée chronique fréquente en Afrique.
\end{abstract}

La prévalence de la toxoplasmose et de la pneumocystose est mal connue (5 à $17 \%$ pour la toxoplasmose et $29 \%$ pour la pneumocystose). Les techniques diagnostiques sont celles couramment connues. La toxoplasmose et la pneumocystose présentent le même tableau clinique que dans les pays développés.

Summary: Opportunistic parasitosis in Africa. Clinical aspects and diagnosis.

Opportunistic parasitic infections are diagnosed more and more often in African patients with AIDS.

Cryptosporidiosis is the most frequent with a prevalence between 7 and $31 \%$. The prevalence of Isospora belli is between 5 and $19 \%$ and that of strongyloïdiasis between 2 and $5 \%$, depending on the authors. These parasitic infections are probably one of the frequent causes of chronic diarrhea in Africa. The preva- lence of toxoplasmosis and pneumocystosis are not well known. (Between 5 and $17 \%$ for toxoplasmosis and $29 \%$ for pneumocystosis).

The diagnostic technics used are those currently known. Toxoplasmosis and pneumocystosis seen in these patients have the same presentations as those in developed countries.
Au point de vue taxonomique, le terme parasite désigne un organisme animal ou végétal ayant une dépendance biologique vis-à-vis d'un hôte dont il tire toute sa subsistance [Crönberg (1)]. Ainsi défini, le parasite peut être un virus, une bactérie ou un champignon. Mais habituellement le terme s'applique à un organisme appartenant au règne animal qui est soit un ectoparasite (par exemple « poux ») soit un endoparasite (par exemple « filaire »). Les parasites peuvent provoquer chez l'hôte des infections subaiguës ou chroniques.

Dans le cadre de l'infection à VIH, le parasite opportuniste est un endoparasite qui chez un immunodéficient est source d'infection aiguë, sévère, récidivante ou généralisée capable d'entraîner la mort alors que chẹez un immunocompétent il ne provoquerait aucune infection généralement grave.

Ainsi définis les parasites opportunistes dont il sera question dans le texte sont le Pneumocystis carinii, le Cryptosporidium, l'Isospora, le Toxoplasma et le Strongylö̈des. Des champignons comme le cryptocoque et l'histoplasme qui à certains égards peuvent être considérés comme des parasites opportunistes ne seront pas repris dans ce texte. Il en sera de même pour certains protozoaires et helminthes

\footnotetext{
* Département de Médecine Interne, Hôpital Mama Yemo, BP 169, Kinshasa 1, Zaïre.

** IMT, Kinshasa, Zaïre.
}

fréquents dans la population normale, particulièrement en Afrique et pour lesquels il n'existe pas de preuve qu'ils jouent un rôle pathogénique particulier chez des patients atteints de SIDA. Il s'agit en particulier du plasmodium, de l'amibe, de l'ascaris, de l'ankylostome et du trichocéphale.

\section{PrÉVAlenCE DES PARASITOSES OPPORTUNISTES}

DANS LE MILIEU AFRICAIN

La fréquence des parasitoses opportunistes dans le milieu africain n'est que partiellement connue. Suivant les études effectuées particulièrement au Zaïre, la fréquence est assez variable d'un auteur à un autre et chez le même auteur suivant les techniques diagnostiques utilisées. En effet, la fréquence de la pneumocystose et de la toxoplasmose par exemple, n'est jamais réellement connue par manque d'infrastructures diagnostiques adéquates.

Quelques données concernant l'Afrique sont résumées dans le tableau $I$.

Comme on peut le voir dans ce tableau, la prévalence de la pneumocystose est mal connue en Afrique. Les seules données valables sont celles de Sonnet et Taelman qui ont étudié 42 patients SIDA africains soignés respectivement à Bruxelles et à Anvers (Belgique). 12 patients soit $29 \%$ avaient une pneumocystose (2). La cryptosporidiose consi- 
TABlEAU I. - Parasites opportunistes.

\begin{tabular}{|c|c|c|c|c|c|c|}
\hline Auteur & $\begin{array}{l}\text { Nb. cas } \\
\text { étudiés }\end{array}$ & $\begin{array}{l}P . \text { carinii } \\
N b .(\%)\end{array}$ & $\begin{array}{c}\text { Cryptosporidium } \\
\mathrm{Nb} .(\%)\end{array}$ & $\begin{array}{l}\text { Isospora } b . \\
N b .(\%)\end{array}$ & $\begin{array}{c}\text { Toxoplasma } \\
\mathrm{Nb} .(\%)\end{array}$ & $\begin{array}{c}\text { Strongyloïdes } s . \\
N b .(\%)\end{array}$ \\
\hline Colebunders 1987 & 106 & - & $23(22)$ & $7(7)$ & - & $5(5)$ \\
\hline Colebunders 1988 & 42 & - & $13(31)$ & $5(12)$ & - & $1(2)$ \\
\hline Henry 1986 & 46 & - & $4(8)$ & $9(19)$ & - & $2(4)$ \\
\hline Sonnet 1986 & 42 & $12(29)$ & $3(7)$ & $2(5)$ & 7 (17) & - \\
\hline Neva 1988 & 41 & - & - & - & - & $11(23)$ \\
\hline Belec 1989 & 93 & - & - & - & $5(5.3)$ & - \\
\hline Pape 1989 & 291 & - & $103(35)$ & $34(12)$ & - & - \\
\hline
\end{tabular}

$\mathrm{Nb} .=$ Nombre $; \mathrm{P}=$ Pneumocystis $; \mathrm{b}=$ belli; $\mathrm{g}=$ gondii $; \mathrm{S}=$ stercoralis.

dérée comme la parasitose la plus fréquemment responsable de diarrhées chroniques a fait l'objet de beaucoup d'études. La fréquence varie de 7 à $31 \%$ selon les auteurs $(2,3,4,5)$. Dans une série haïtienne, la fréquence de cette parasitose a été trouvée à $35 \%$, légèrement supérieure à celle trouvée à Kinshasa (6). Isospora belli a aussi suscité beaucoup d'intérêt comme protozoaire le plus fréquemment retrouvé comme opportuniste. Sa prévalence serait de 5 à $19 \%$ suivant les auteurs $(2,4,5,3)$. Dans la série haïtienne, sa prévalence est de $12 \%$ (6). La toxoplasmose comme la pneumocystose ont été insuffisamment étudiées en Afrique faute d'infrastructures. Une étude de Bangui donne une fréquence de $5 \%$, tandis que celle de Sonnet et Taelman révèle une fréquence plus élevée $17 \%$ (2). La strongyloïdose est considérée comme l'helminthiase opportuniste la plus fréquente. Cependant certaines études réalisées en Afrique ont été décevantes avec une prévalence plutôt faible de 2 à $5 \%$ des patients SIDA infectés $(3,4,5)$. Mais dans une recherche effectuée à Kinshasa par Neva suivant une technique mieux appropriée, la prévalence de cette parasitose a été de $23 \%$ dans une série de 41 patients SIDA et de 18,2 \% dans une série de 71 patients $\mathrm{VIH}$ positifs (8).

\section{DiagnostiC Des PaRAsitoses OPPORTUNistes}

Si la prévalence des parasitoses opportunistes varient d'un auteur à un autre ou d'une région à une autre, c'est dû en partie aux moyens disponibles et aux techniques appropriées pour asseoir le diagnostic.

Pneumocystis carinii. La technique la plus utilisée est la bronchoscopie avec lavage broncho-alvéolaire. Elle peut être enrichie par la technique de liquéfaction induite de l'expectoration (induced liquified Sputum). Plusieurs techniques de coloration sont ensuite utilisées pour révéler le parasite. Les meilleurs sont probablement la coloration de May-Grünwald Giemsa, la coloration de Gram-Weigert et l'imprégnation argentique de Musto (9). La biopsie trans- bronchique et la biopsie pulmonaire, agressives, sont parfois utilisées.

Cryptosporidium. Ce parasite se recherche habituellement dans les selles. Des prélèvements biopsiques intestinaux sont aussi une voie de recherche bien que les résultats ne soient pas meilleurs que l'examen simple des selles. Il existe aussi plusieurs techniques de coloration des préparations des selles pour la mise en évidence des oocystes de Cryptosporidium. La technique de Ziehl-Neelsen est la plus utilisée.

Toxoplasma gondii. Le diagnostic du toxoplasme se réalise soit par séro-diagnostic (recherche d'anticorps antitoxoplasme dans le sang ou dans le liquide céphalorachidien), soit par CT-Scan avec ou sans injection intraveineuse d'un produit de contraste. En post mortem, l'autopsie permet des prélèvements pulmonaire, cérébral, hépatique, cardiaque et autres pour un diagnostic histologique. Le séro-diagnostic est de peu d'intérêt. Dans les pays et les institutions où cette technique est disponible, le CTScan est la technique de choix.

Isospora belli. La mise en évidence facile du parasite se fait soit par l'examen direct des selles soit par la technique de Kato (9). Des biopsies intestinales permettent aussi de révéler le parasite.

Strongyloides stercoralis. La recherche du parasite se fait soit dans les selles par un examen de selles direct ou suivant la méthode de Neva (10), soit dans les expectorations (recherche des larves). En plus, un «Skin test antigens » pour Strongyloides est une voie d'approche diagnostique (10).

\section{Clinique}

Pneumocystis carinii (Pneumocystose). L'expression clinique d'une infection à Pneumocystis carinii est une pneumopathie souvent non dyspnéisante avec comme symptôme dominant une toux non productive associée ou non à la 
présence des crépitements pulmonaires. La fièvre, l'amaigrissement et la dyspnée constituent avec la toux un tableau clinique d'une infection sévère.

Cryptosporidium (Cryptosporidiose). Ce parasite serait responsable de diarrhées profuses et récidivantes chez les immunodéprimés. Certaines atteintes des voies biliaires seraient aussi dues à Cryptosporidium.

Isospora belli (Isosporose). La diarrhée est l'expression clinique majeure d'une infection à Isospora belli. Une infestation sévère peut entraîner une diarrhée mortelle.

Toxoplasma gondii (Toxoplasmose). Une toxoplasmose neurologique est la forme classique d'une infection à Toxoplasma gondii chez les sidéens. Elle s'exprime par des céphalées, une dépression, une faiblesse générale, des déficits neurologiques focaux, des convulsions et le coma. Mais il existe des infestations pulmonaires, hépatiques et cardiaques.

Strongyloides stercolaris (Strongylö̈dose). Ce parasite provoque surtout des diarrhées abondantes. Mais il peut aussi entraîner une septicémie bactérienne, une méningite, une pneumonie ou un ileus paralytique.

\section{Conclusion}

Les parasitoses opportunistes sont sûrement des complications majeures du SIDA en Afrique. Leur connaissance doit être approfondie pour une meilleure prise en charge des patients.

\section{RÉFÉRENCES}

1. Crönberg et al. : Maladies infectieuses. Masson, Paris, 1988, $464 \mathrm{p}$.

2. Sonnet J., Taelman H. : Clinical and biological profile of African AIDS. A study of 42 patients. Clinical aspects of AIDS and AIDS-related complex. Oxford university Press, 1986, 78-89.

3. Henry M. C. et al. : Parasitological observations of chronic diarrhoea in suspected AIDS adult patients in Kinshasa (Zaire). Trans. R. Soc. Trop. Med. Hyg., 1986, 80, 309-310.

4. Colebunders R. et al. : Persistent Diarrhoea Strongly Associated with HIV infection in Kinshasa, Zaïre. Am. J. Gastroenterol., 1987, 82, 859-864.

5. Colebunders R. et al. : Persistent diarrhoea in Zairian AIDS Patients: an endoscopic and histological study. Gut., 1988, 29, 1687-1691.

6. Pape W. J. et al. : Treatment and Prophylaxis of isospora belli infection in patients with the acquired immunodeficiency Syndrome. N. Engl. J. Med., 1989, 1044-1047.

7. Belec L. et al. : Manifestations Neurologiques et Psychiatriques du SIDA en République Centrafricaine. Bull. Soc. Pathol. Exot., 1989, 82, 297-307.

8. Neva F. A. et al. : Projet SIDA Perinatal Study, 1988 (en préparation).

9. Datry A. : Diagnostic des parasitoses et mycoses. SIDA, Infection à VIH, aspects en Zone Tropicale. UREF, 1989, 218-223.

10. Neva F. A. : Biology and Immunology of human strongyloidiasis. J. Infect. Dis., 1986, 153, 397-406. 